Article

\title{
Consuming Production: Anime's Layers of Transnationality and Dispersal of Agency as Seen in Shirobako and Sakuga-Fan Practices
}

\author{
Stevie Suan \\ Center for Global Education, Doshisha University, Karasuma-Higashi-iru, Imadegawa-dori Kamigyo-ku, \\ Kyoto 602-8580, Japan; steviesuan@gmail.com
}

Received: 8 May 2018; Accepted: 28 June 2018; Published: 16 July 2018

\begin{abstract}
As an alternative reading of anime's global consumption, this paper will explore the multiple layers of transnationality in anime: how the dispersal of agency in anime production extends to transnational production, and how these elements of anime's transnationality are engaged with in the transnational consumption of anime. This will be done through an analysis of Shirobako (an anime about making anime), revealing how the series depicts anime production as a constant process of negotiation involving a large number of actors, each having tangible effects on the final product: human actors (directors, animators, and production assistants), the media-mix (publishing houses and manga authors), and the anime media-form itself. Anime production thus operates as a network of actors whose agency is dispersed across a chain of hierarchies, and though unacknowledged by Shirobako, often occurs transnationally, making attribution of a single actor as the agent who addresses Japan (or the world) difficult to sustain. Lastly, I will examine how transnational sakuga-fans tend to focus on anime's media-form as opposed to "Japaneseness", practicing an alternative type of consumption that engages with a sense of dispersed agency and the labor involved in animation, even examining non-Japanese animators, and thus anime's multilayered transnationality.
\end{abstract}

Keywords: anime; transnationality; transnational animation production; performance; media-form; dispersed agency

\section{Authorship and Agency in Anime Production: A Transnational Perspective}

The approach to anime as a media consumed transnationally is often one where the audience is seen as consuming "Japanese culture." In academia (which is its own type of consumption-production), an extension of this is revealed in some analyses that focus on a particular work's commentary on Japanese society. It is often a film that is taken up as the subject of inquiry, with the director the person the work is attributed to. A set of famous directors occupy the majority of the focus: Hayao Miyazaki, Mamoru Oshii, Satoshi Kon, and, more recently, Mamoru Hosoda and Makoto Shinkai. ${ }^{1}$ Such a concentration on film is reflected in the tendency to elevate these directors to the status of auteur, even though the problems of auteur theory from film criticism are widely acknowledged. In consideration of this, there is often an implicit understanding that there are other agents involved in the production, but researchers continue to utilize the singular director as a type of short-hand for an agent which orchestrates the major decisions of the production. Here "production" becomes less of a process as the focus switches to the "producer" as the central, authorial agent. In a sense, such an approach makes the film product become the director's "speech-act", so to speak. This allows for an

1 To a lesser extent this extends to Isao Takahata and Katsuhiro Ōtomo as well. 
analysis of the anime work in question as producing a commentary, a commentary that is often seen as addressed to Japan and read as focusing on the topic of Japanese society.

Here I do not mean to say that directors should not be given recognition, or that they are not involved in many levels of the production (for which Miyazaki is notorious). Rather, I want to highlight how this tendency of attribution to some singular agent reveals a tendency towards certain conceptions of authorial agency, creative and cultural production, and methodological approaches to interpreting (and consuming) anime. When considered in the context of transnational consumption, we find the same methodological approaches common in analyzing anime as a commentary on Japanese society by directors: anime is "Japanese culture" consumed outside of Japan, making anime works a speech-act about Japan, because, as is commonly thought, anime comes from Japan. This produces a conception of transnationality whereby people consuming anime outside of Japan are consuming Japanese products, which is less transnational (across borders, operating beyond the received notions of nation-state) and more inter-national (two distinctive nation-states engaged in cultural exchange, Iwabuchi 2010), producing a sense of "inside-outside" that is relatively neatly defined-in this case, consuming Japanese media (produced inside of Japan) while the consumers are outside of Japan. Thus, there is a sharp distinction seen between production (authored by directors in Japan) and consumption (inside vs. outside of Japan) that overlaps with the idea of the inter-national (distinctive nation-states), which might be overcome by engaging with both production and consumption to explore the transnational dynamics of anime.

This is not a disavowal of reading anime as a commentary on Japan or the contributions such scholarship has made. Nor is this a denial of the importance of the nation at play here. Rather, this is a suggestion for exploring an alternative approach that more fully engages with transnationality. For instance, while it is widely accepted that anime is a transnationally consumed product, and it is generally acknowledged in academia that anime has a history of transnational production, the implications of these layers of transnationality are not often explored. This is important to consider as much of anime's transnational labor is for the animation, which is the basis of the very media product itself: anime is animation, making the results of the animators' labor a significant portion of what we actually receive as the final work. We might connect the lack of exploration to the above-mentioned tendency to emphasize directors (who operate in Japan) over animators (who operate across national borders), which also relates to the framework of the inter-national rather than the transnational. As such, it can be productive to consider a more inclusive view of anime's transnationality - that is, accounting for the dispersal of agency in anime production, how this extends to transnational production, and how (and if) these elements of anime's transnationality are engaged with in the transnational consumption of anime.

In order to explore the dynamics of agency in anime production, in this article I will conduct an analysis of the anime Shirobako (2014), which details a number of processes of anime production as the central narrative of the series. Broadly, this analysis is also done in reference to Ian Condry's (2013) ethnographical work on the anime industry in Tokyo, which he describes as a "collaborative creativity" that involves not just typical creative producers (animators, directors) but even corporate executives. In terms of methodological approach, this article is underpinned by Bruno Latour's (2005) "actor-network theory." I use "agency" here as an adapted version pulled from Latour's work, where an "agent" performs actions, "making some difference to a state of affairs, transforming some As into Bs through trials with Cs" (pp. 52-53). Agents that exercise such capacities are not limited to human actors but include a whole host of non-human actors that work with/on humans, including various materials, technologies, and anime's media-form itself-that is, the repeated patterns utilized that distinguish anime as a particular type of media product.

Because of the relationship between the multitude of human and non-human actors, each working with, on, and through one another, one can trace an intricate web of actions that affect the final product, often in directly discernible ways (e.g., the narrative, the animation, etc.). As such, instead of a single agent in one location who is the source that orchestrates the production, there is a dispersal of 
agency, a "network" of actions from multiple agents (at different times, in different places) and the negotiations that arise from their mutual engagement that result in effects that we see in the anime product. This does not mean that an anime work cannot sustain itself as a commentary on a topic, or cannot be read as exploring an issue, but that the source of that exploration becomes complicated, as does the context with which it may be engaging. As a self-reflexive exposition of anime production, Shirobako depicts such a web of effects. In the analysis that follows, I will show that anime production involves a large number of actors with differing degrees of control, each one involved in diverse developments that result in a constant process of negotiation, the outcomes of which are tangible in the final product (the anime itself) and can (and do) occur transnationally. Anime production thus operates as a network of actors whose agency is dispersed across a chain of hierarchies, making attribution of a single actor (human or non-human) as the agent who addresses Japan (or the world) difficult to sustain.

Furthermore, Shirobako, as an anime, invites (if not enforces) an alternative approach to consuming anime, less as a commentary on Japan per se, and more on the anime industry itself. The series provides a sharp focus on the labor involved in anime production, with a particular emphasis on the animation: much of the drama of the series is motivated by issues involving the animation itself (though other elements, such as story-boarding, script-writing, and voice-acting are prevalent). When these animation production issues are resolved, many times they will display the intradiegetic animation at the end of the episode, the sakuga (a complex animated sequence), often lasting just a few seconds, but revealing the fruits of the labor (and its conflicts) that were just witnessed as the drama of the episode. In this way, the series forces the viewer to focus on the sakuga sequences as the product of labor by many people. These sequences are all from the intradiegetic anime the studio is producing, but one which we (the viewers) do not know the details of the narrative. ${ }^{2}$ This type of consumption of the animation sequence itself, estranged from narrative, resembles the type of consumption practices employed by a particular type of fan: the sakuga-fan.

While I have glossed sakuga above as "a complex animated sequence," the very term "sakuga" is slightly ambiguous in Japanese. At its most simple, it is "images that are made" and is usually associated with key-animation (officially known by the separate term of genga-key-frames that are not colored and do not include in-betweens) but sakuga can also mean the final cut of an animated sequence. As such, the term implies that it is merely one part of a larger production process and holds the potential for an ambiguity of attribution to multiple agents in its production while still attributing credit to a key-animator. Terminology aside, it is the sakuga part of anime production that is the central concern of sakuga-fans. They are interested in these key sequences as the focal point of production. While not ignoring elements like narrative, character design, or voice-acting, sakuga is what they concentrate on.

As such, in the final section, I will transition into how sakuga-fans tend to focus on form and the materiality of the medium of animation as opposed to "Japanese society." The products of their consumption practices are published on the internet, making the engagement with their work transnational, as it is available freely on the internet, across the world. I will examine how, based on English-language YouTube and blog posts by such fans, they present an alternative type of consumption that engages with a dispersed sense of agency regarding animation, the people involved, and how they also examine non-Japanese animators (and thus anime's transnationality). In fact, this focus on the animation and its production sets up an alternative type of viewing that generally disregards narrative in favor of the craft of animation and the people who labor behind it. Interestingly, these transnational fans who focus on form also seem concerned (and critically engaged) with the labor of animators and their harsh working conditions inside (and outside) Japan.

2 Though, it should be noted, the two intradiegetic anime produced by Musashino Animation were later released as one-episode OVAs. 


\section{Shirobako: Analysis}

What makes Shirobako relatively unique in the TV anime world is how it brings the dispersal of agency in animation production to the foreground. There are precursors to Shirobako, such as Otaku no Video (1991), which details the transformation of a college student into an otaku who then creates two otaku media companies, and Bakuman (2010), a series about an aspiring manga creator and their relationship with the anime industry. However, Shirobako focuses almost entirely on the anime industry, exploring the finer details of anime production itself. Centering around the production assistant and administrator Aoi Miyamori, Shirobako follows her role at the fictional anime studio Musashino Animation, and includes various characters heavily inspired by those in similar roles (in different studios) in the real-life anime industry. In the background, Miyamori's four friends from her hometown have also joined the anime industry in different roles (2D key animator, 3D animator, script writer, and voice-actress), and they dream of working on an anime together. The narrative of the series follows the production of two different intradiegetic anime (Exodus! and The Third Girls Aerial Squad) and the drama that occurs during the production process of TV anime. As such, Shirobako provides a convenient object of analysis with which to examine the workings of the anime industry and exactly who (and what) is guiding the production, making decisions, and producing effects on the final product. However, because of the self-reflexive mode of address (an anime about making anime), the work should be approached with a strong dose of skepticism. Its self-representation should not be considered the final word on the status of the industry and can easily overly dramatize or misrepresent the actualities of the work place.

Though acknowledging that this is not necessarily an accurate depiction of the anime industry, the form of the presentation of Shirobako (an anime about making anime) constructs a self-referential account of the anime industry while also offering some evidence for the claims about distributive agency in anime production. Through an analysis of key sequences of the series, I will (1) discuss how there is a production hierarchy where various roles exert different degrees of control over the creative process; (2) explore the agency of the materials involved as the animators and directors struggle with elements of cel-animation and its mixture with CG animation, and briefly depict the differences between manga and anime production/industries; (3) detail how the media-form constrains the production: different expectations for the product, the types of facial expressions used, and the structure of the narrative (action-oriented climaxes); and (4) provide some brief information on the transnational production involved in Shirobako — something unacknowledged in the series-and discuss how this manner of dispersed agency in anime production frees anime to acknowledge its transnationality rather than emphasize its exclusive nationality. For ease of explanation, these have been divided into distinct sections. However, it is important to note that many of these are interconnected, such as when the capabilities of the medium (cel animation) allows for a complex division of labor, which affords for (transnational) sub-contracting, and additionally, can affect where and when certain types of animation are employed in the narrative.

\subsection{Negotiated Decisions}

Let me begin with the production hierarchy, which, as noted before, is often conceived with the directors and top-level producers at the top of the chain of command-those making the decisions, so to speak. Even if this is expanded, anime is so often seen from the perspective of a type of "creative industry" in which animators, writers, designers, voice-actors, and musicians get much of the spotlight (and credit) for a production. However, Shirobako consistently undermines this conception of the hierarchy of decision making power in anime. In Shirobako, an administrator takes center stage and is shown as integral to the anime production process, part and parcel of this creative industry. This is evident in the position of the main protagonist, Miyamori, who performs the role of "desk", a production assistant who organizes and relays information and materials between animators and top-level staff but also works towards solving problems that have tangible effects on the final product of the animation. However, the administrators are not the only positions whose actions are shown 
to affect the final product. A whole slew of roles is featured, such as general managers, in-between animators, sub-contractors, freelancers, colorists, editors, sound engineers, publishing executives, and manga authors. Indeed, there are so many characters that names and position titles are often extra-diegetically inserted next to the characters in every episode, something rarely done with such frequency in anime. Furthermore, many of the characters switch roles between the first and second TV anime produced at Musashino, moving into different positions, showing how each particular production can be executed very differently, despite including most of the same people.

Because of the large group of people involved, the complex hierarchies of production, and the diversity of technical, administrative, and financial expertise necessary for the successful planning and implementation of a production, the series has plenty of points of conflict when nothing runs smoothly. Many of the conflicts featured disrupt the conception of the singular vision of a director, with multiple agents involved, including supposedly minor roles such as production assistants sitting in on (and influencing) important meetings with the top-level producers. This also includes the enforcement of conducting the labor itself. In episode 5, for example, the "desk" for the first TV anime (Exodus!), Yutaka Honda, forces the director to work by comically locking him in a cage to finish the storyboards, showing how the director is the one who produces the story-board, but the enforcement of the actual enactment labor is coming from other roles.

These conflicts also involve non-human actors, who often receive close-up shots of the specific materials or sequences that display their use (or malfunction): servers that transfer data unexpectedly go down, causing chaos; cars (and their drivers) that can deliver the goods at the speed necessary; and, of course, the cels, papers, pencils, and computers used to make the animation, which each must be worked with to produce an anime. Storyboards are important to consider in this light as well. The storyboards' creator might be seen as another source of authorship, as they are adaptations of a script which will be used as a central reference point by the animators and editors for the rest of the production. It should be noted that, in reality, it is not always the case that the director will do the storyboards. Sometimes it will be the episode director, or another member of the staff, and storyboards are finalized in meetings with many members (Condry 2013, pp. 10-13). While credit should not be denied to the storyboard creator, the storyboards themselves also become an agential actor, as they guide much of the production from that point onwards. As Condry notes, while storyboards are filled with details and instructions from their author(s), there are multiple different interpretations that can come from the storyboard object (2013, p. 48). Such issues are worked out in meetings (pp. 10-13), but are also left up to those with the skills to interpret and produce a refined section of the production based on the storyboards (p. 12). As such, the storyboards are important actors in anime production that animators (and other staff) must grapple with (often at a distance from the producer of those story boards), working with them to perform their various roles in the production. This is addressed in episode 15, when the director meets with the animators to explain what types of images he wants from his storyboards, and extradiegetic comments state that the animators have to make more polished images from the rough images given to them on the storyboards. In a sense, the animators are negotiating with the storyboard itself, as they must make certain creative decisions from the instructions detailed on the storyboards, adding such interpretations into the animation of the final product we see.

Further negotiations about how to execute the storyboards come from still more workers with multiple chains of command. This continues to create conflict down the line. While Miyamori and another production assistant argue over whose fault certain problem are in episode 5, they understand that everyone must suffer the consequences, and it is only through the cooperation of various workers that the issue can be fixed. Although there is a clear chain of instruction, with directors and producers at the top, Shirobako makes an effort to show how collaborative the creative process actually is, revealing how even the production assistants affect the final product in their daily negotiations and decision-making. 
While the effect of animators in particular will be addressed below, there are also other positions in the production process that are shown to undercut, interrupt, and affect the final product. These even come from outside of the studio itself, problematizing the view of even a single studio as the source of a product. Shirobako exposes the importance of relationships within the larger industry (including anime, manga, and other related products), with decisions often occurring outside of both the Musashino studio and a formal business environment (e.g., offices, conference rooms). For example, in episode 12, producers from Musashino informally discuss with an executive from a publishing company about the possibility of their studio getting the contract to produce the hit manga series The Third Girls Aerial Squad over a game of mahjong. Ultimately, Musashino gets the contract, displaying how important a network of contacts within the industry is for a studio to stay relevant with in-demand material to base their productions on, showing how integral other media are for the anime industry. It also reveals how anime studios "chase" those who hold the rights for such source material. Anime studios appear dependent on source materials (they cannot always produce "original" works) and are in a sense under the control of the companies and their executives, who are higher up on the hierarchy of production. Indeed, Condry also notes the importance of various executives outside of what is normally considered production staff in multiple different anime productions that he observed (2013).

While the contract for The Third Aerial Girls Squad comes from the company that publishes the manga, this is a bit of an oversimplification of the actualities of anime production. In reality, anime production since the mid-1990s has been funded by a production committee made up of a number of different companies that each contribute a small portion of the investment to produce the anime but maintain certain portions of the rights for the intellectual property. Despite this discrepancy, the interaction with the publishing company-presumably a stand-in for the production committee-produces engaging drama and displays some of the realities of how dispersed agency operates in anime production, even at the top of the chain of command. For example, the importance of voice-actors is highlighted, especially in episode 14, when producers and talent agents (as well as the uninterested representative of the publishing company that owns the rights to Aerial Girls Squad) argue over how different variables-such as the voice-actor's role as idols or their current fan following, as well as their actual capacity to play the given role-are considered when casting for the anime. The inclusion of the representative from the publisher highlights their importance in the hierarchy of production, but also the role of the anime as part of a larger media-mix, where the anime is one product among many with a different capacity to draw in fans-voice-actors are one of these avenues, an aural component to anime that is not physically present in manga.

The difficulties of such a production hierarchy are revealed throughout the series. For example, in episode 13, when the character designer is changed, the producers worry that the publishers might not be happy (and thus they may lose their contract), as they may have chosen the studio based on the staff from their previous production. There are also crucial moments when the publication company executives and/or the author of the manga step in and reject the work produced by the anime studio. This includes forcing changes to character design as in episode 16, even though Musashino was well into the production process based off the designs. Such influences become extreme in episode 23 when we learn that, due to poor communication between the publishing company's representative and Musashino studios, the manga author rejected the storyboards by the anime studio for the ending of the series (which would continue past the manga's current narrative developments). Though they received a preliminary agreement from the manager of the manga author at the publisher and proceeded quite far in the production (including the voice-acting), they are rudely requested to re-do the final episode. On top of this, the publishers do not allow Musashino studio's staff to meet with the manga author directly, causing a large problem.

Such instances display the actual authority of the publishers (stand-ins for the production committee) over even the top-level producers and director of the anime. In this way, Shirobako establishes a chain of capacity for decision-making, with the publisher/production committee at 
the top, followed by the director and producers. However, this is always complicated by the actual processes of production, in which episode directors and animation supervisors, as well as the animators themselves, make important decisions, or when the production assistants solve various problems and make suggestions (or force higher-ups to work) that result in tangible effects in the final product, examples of which I will examine below. As such, Shirobako provides an account of anime production in which there are layers of decisions upon decisions, in which there is the assertion of agency at multiple levels within the chain of command, each action adding (or subtracting), adjusting, and transforming the results we see in the final product. Shirobako makes us recognize the different shifts that occur due to each actor (human and non-human) in the production process, each having an effect on the final images and sounds, seen and heard. It displays the struggles behind each frame and the labor behind the animation, producing a multilayered take on anime production.

\subsection{Media-Mix and Materiality}

Because anime production is so often part of a media-mix, anime producers must work in conjunction with the publishers/production committees to maintain a certain degree of similarity (or planned divergence) with the other media. This means that there may be conflicts between the different producers of the various media, a conflict that is not always based on the differences in a "creative vision" of the agent(s) of production but a divergence that stems from the diversity (or lack thereof) of materials involved in their respective processes. As such, a large degree of agency must be negotiated in the process, as directors and producers must tackle not just the executives that own the intellectual property but the challenges of working with and in the mediums that the different media of the media-mix are produced in.

This has wide-ranging consequences. For instance, in episode 20 there is a meeting discussing how to approach the ending of the anime of Aerial Girls, in which the director, producer, top administration staff, and the script-writer brainstorm about possible directions for the narrative, as the anime's airing will outpace the manga's publication (a common occurrence in anime adaptations). The director points to how in manga it is easier to change directions of the story than in anime. Debating on whether the protagonist in Aerial Girls should not fly, continuing the trauma she received in a previous episode that kept her from piloting (a regular trope in mecha anime/manga), or should rise to the challenge at the end and fly, overcoming her crisis. In the current stage of the manga, the pilot is left grounded, but the staff argue that in anime, the expectations are different, and the pilot must fly in the end to make a satisfactory ending.

While there is a chain of command here, it is clear that there are multiple different actors, each exerting varying degrees of agency throughout the process. This gets further complicated as the agreed plan of letting the pilot fly is directly undercut by the meeting with the manga author of the Aerial Girls in episode 23. The anime director makes his case that the protagonist pilot should fly, because, like in his life, they continue to fight because of their compatriots, because of those working hard around them. The manga author replies that, for the anime director, the anime production team is like the Third Aerial Girls Squad, to which the director agrees. However, the manga author sees it differently, because each of the girls in the aerial squadron is a metaphor for the problems that plague him personally. While entertaining the myth of the lone manga author here (in truth, manga authors often have assistants and work very closely with editors), a difference between mediums is revealed: theoretically, manga can be made by one person with pen, paper, and publishing technology (print or digital) who has developed a certain set of skills (e.g., drawing, developing narratives and characters, paneling, etc.), whereas anime generally involves a large group of people, each with various specialized skills (e.g., animator versus script-writer versus voice-actor) and access to different technologies (different both from the technology and materials of manga, as well as in terms of diversity of technologies used). This difference in medium-specific labor practices develops into a difference of group dynamics, which is further extended into the respective approaches each creator (manga and anime) takes when addressing the problem of how to continue the narrative. However, 
this difference becomes productive through the collaboration of the director and manga author: it is the anime director's interpretation of the manga author's approach that sparks a renewed understanding of the pilot's character, one which the manga author then affirms. The director then proceeds to suggest a way to provide an internal motivation for the pilot, and through their interactive brainstorming, produce a satisfying solution to this problem of the narrative, one which satisfies the demands of anime's narrative conventions and the manga author's convictions. Furthermore, they actively work together, a productive outcome of conflict that results in a similarity between the anime and manga versions of the work.

Here we see how, despite the apparent closeness of manga and anime, there are divisions that the specific mediums (comics vs. animation) produce. With this in mind, from the current approach of "actor-network theory", the materials used in production become actors, the differences in mediums affecting the type of production processes and their group dynamics (as in the discrepancy of interpretations between anime director and manga author). More to the point, the above described interaction between director and author exposes how human agents must work with the affordances of the mediums, in concert and in tension with the material, to conspicuously produce an effect of similarity between manga and anime and invoke a recognizable relation between them that synergizes the media-mix (cf. Steinberg 2012).

This problem of maintaining a relation between anime and manga and their distinctions in the materials of production occurs even in areas that would be supposedly simple to solve. For example, during the character-design phase for the production of Aerial Girls in episode 13, the character designers explain the difficulty of designing a character from the 2D manga for the anime, which has to work in three dimensions in animation. As Thomas Lamarre has detailed, character design is itself a crucial element of anime (and manga's) engagement with the moving-image (what Lamarre calls "soulful bodies"), both in the medium of (limited) animation and as it relates to the character's ability to move across media (the media-mix) (Lamarre 2009, pp. 200-4; see also Steinberg 2012). As such, the character designer must twist and morph the designs that come from one medium (comics) to work with the affordances of the materials at hand to adapt them for another medium (animation), maintaining a clear resemblance to the manga (referring viewers to the manga product as well as other media) while still allowing for the operability of the character designs in animation. In this manner, character design adaptation is another process of negotiation between material and human actors.

Yet, there is another dynamic at play in the adapting of character designs from manga to anime. This is referenced in episode 13, when Rinko Ogasawara, the previous animation director, notes how in the process of imitating the source manga, repeating the characters over and over, eventually they become the animator's own characters, and if this does not occur then the animator cannot be a character designer. Her statements thus frame producing an adaptation as a problematic of reiteration, bringing attention to an important tension between repetition and variation, in which reiterations somehow eventually produce change (Suan 2017b). In these processes, there is not only the operations of working with certain materials to produce the work but also an active engagement with a history of conventions from earlier anime works: anime's media-form.

\subsection{Anime's Media-Form}

Before going further, let me better explain what I mean by media-form. Concisely, anime's media-form is the repeated conventions that we see in anime, i.e., what makes anime recognizable as such - the reiteration of these conventions producing the resemblances that we see in anime works. Such conventions are myriad, from the character designs to the voice-acting styles, from the narratives to the character expressions and animation techniques used, all with a history. These conventionalized elements become what we expect out of anime as a particular media product, as new anime works have a relation to earlier works through the repetitions that sustain theses expectations. This has developed into a system of such conventions, such as Azuma Hiroki's database (Azuma 2009), even as the conventions shift over time. Anime's media-form is thus performatively constituted by the enactment 
of large quantities of these conventions in the animated productions we call anime (Suan 2017b). The term "media-form" is used to distinguish itself from genre (which is often used to imply works that employ particular thematic tropes: romantic-comedy genre vs. robot genre) or style (which is often used as something individualized: a particular author's or artist's style), as the conjoined words "media-form" highlight formal notions while emphasizing how, following Lamarre (2009), anime should not be separated from its performance in the medium of animation (Suan 2017b, pp. 65-66). It is this performance of the anime media-form that is the very subject of the drama of Shirobako and is what makes Shirobako itself recognizable as an anime.

What this means, then, is that anime's media-form is another element at play here that is constraining and structuring the production process, something that restricts the staff within certain boundaries (what is expected of the anime media-form) while also giving them a point of departure, thus making them the agents of anime production. Let me provide an example. In episode 12, the top-level producers and administrators discuss what to do for an important scene at the climax of the Exodus series. They argue over the feasibility of producing the scene the director wanted involving a herd of horses. Animating such a scene would be complex and difficult to produce in terms of the technical skill of the animators, as well as the time it would take to produce it. A suggestion by one of the producers was to either do the scene in 3D (which would be difficult because the 3D animators were already overbooked and busy) or adjust the story-boards to show scenes that pan over the horses and do not show their legs moving. This is harshly objected to by the managing production assistant Honda. He argues that this is a climactic sequence and cannot have shoddy animation sequences (sakuga), insisting on the importance of the climax for the success of the series itself. Indeed, even Shirobako follows the patterns they discuss in the show: the episodes at the climax, in particular, episode 23, feature complex, action-oriented sequences as the director sneaks into the publisher's office building, comically performing game-like martial arts moves to defeat a string of "bosses" (the executives and managers at the publishing house) who try to keep him from meeting the manga author.

In such a conflict, there are multiple forces at play here that drive the decision-making process: the time, staff, and budgetary restrictions, but also the expected structure of the anime series, an expectation built from previous anime, which demands complex and exciting animated sequences to ensure the success of the series. The director was working towards the latter, while the producers must provide a solution for the former. Ultimately, it is Miyamori who delivers a viable solution which satisfies both requirements-a solution that was itself suggested from a famous animator who is entirely outside of the company - that is, using the in-house veteran animator Shigeru Sugie to animate the horses. However, even this final animated sequence is achieved only through a team effort. Due to the time-restraints, Sugie (who usually works on children's animation) must draw rough sketches that are then to be cleaned up by other lead animators. In fact, the two animation supervisors actively volunteer to do this job, as they want to learn from his techniques and participate in this part of the production. Here, we not only see the multiple layers of laborers' work that go into a single animation sequence (key-animation, clean-up, and in other sequences, coloring and then editing), but it also reveals how important building on the expectations from anime performances are for the production process.

Because of the importance of reference to other examples of animation, there is a certain delimiting range based on previous examples that structures the type of animation considered acceptable in anime, highlighting how particular the performance of anime's animation is. We can see this in another example when the junior animator Ema Yasuhara becomes overcome by fears of producing poor animation for a sequence involving a cat. Her fears are visualized as the image of a cat with sharply defined lines that "devolves" into a squirmy ball that loses its distinction as a "cat." In these sequences in episode 8 , it explicitly shows there is a clear preference for a particular type of animation, straying away from amorphous shape-shifting objects towards more "stable" images which remain recognizable. While in this context it is a display of fear for reduction of quality, certain types of "art animation" purposefully produce destabilized images or have character outlines that "vibrate" and 
are inconsistent. Yasuhara's fears visualized as such reveals a general tendency within anime to shy away from certain types of animating of objects (humans, or cats, etc.) in favor of more rigidly defined stylistic boundaries. To help Yasuhara get over her fears, general animation supervisor assistant, Yuka Iguchi, takes her out for a walk and instructs Yasuhara to look at previous examples of animation and to copy them, to "learn by copying", implying that animators are building their skills from previous examples but also stressing the importance of copying and repetition, as well as relations to previous examples of anime history in the production of contemporary anime-something that is somehow both beyond their control (in the past, outside of their immediate decision making) and that they can also contribute to (through copying and their own productions).

This is also evident in a story-arc that occurs over episodes 4-6 when there is a dispute between a 2D animator, a production assistant, and a 3D animator. Here, the 2D animator (Ryūsuke Endō) abruptly withdraws as animation director, because the 3D director had already finished the animation of an explosion in CG, which the 2D animator wanted to draw. Feeling as if 3D was becoming favored, and due to the poor relaying of information by the production assistant, the 2D animator becomes angry and withdraws. Ultimately, the issue between 2D and 3D animation is solved in episode 6 by a visit to an exhibit of a fictional anime (based off of the 1980 anime Space Runaway Ideon), and the two animators bond over their mutual affection for that earlier series. This is one of the many sequences where they show how the animators, as well as other staff, were (and still are) fans of anime, active consumers who have devoted themselves to its production. This feedback loop of consumer (fan) to producer (animator), in which consumed anime informs produced anime, is further emphasized in Shirobako in sequences in which the anime animators observe themselves, other characters, or creatures to animate them by this reference. Because the show's mode of address is in anime's media-form, the characters are observing the anime world to (re)produce anime-it is literally self-referential.

This brings me to one of the most recognizable elements in anime that exposes how "external" (and thus imposed, learned, and performed by the animators) anime's media-form is to the production process: anime's conventionalized facial expressions. The performance of facial expressions in Shirobako becomes an important plot-point during episode 3, when they have to re-cut a certain segment because the "images" (e) are "losing" (maketeru) to the voice-actress' performance (shibai). The general animation supervisor assistant, Iguchi, must redraw the character expression, making it more expressive. This must be done in a timely manner, and so Iguchi concentrates on this particular expression, spending all night drawing the images, repeating to herself the sentence "I knew it", the lines that the character must say during the enactment of that pained expression. This is a general practice in animation, in which an animator works through an expression to get the timing correct, or views their own face making that expression-a practice that then makes the animator take on the actions of the animated character, and the character becomes imbued with the actions of the animator (Kim 2015). However, what is important to consider in anime is that character expressions have to carefully balance the repeated conventionalized codes, what Donald Crafton calls "figurative acting" (Crafton 2013; Suan 2017a), with the individualized expressions specific to that character's emotions (what Crafton calls "embodied acting"). Often, anime characters tend towards a stricter repetition of these codes, and indeed, the pained expression Iguchi is animating for the character Aya in Shirobako's interdiagetic anime Exodus! is one that is performed very similarly by characters in many other anime. In such sequences, characters (often female) lower their head; their eyebrows become upside-down arches that move closer to the center of their head, and their eyes transform between lightly arched lines, to squinted eyes, and then become filled on the side with tears; as the head moves (often in a diagonal, then circular motion), the tears disperse into water droplets. For example, though this post-dates Shirobako, the character expression by Akko in episode 6 of Little Witch Academia (2017) is 
performed in a very similar manner ${ }^{3}$-an overdramatic expression that is regularly repeated, not in the exact same manner, but in the general model that is adhered to across multiple distinct anime productions (Suan 2017b, 2017a). What is intriguing here is that Iguchi herself is an anime character, thus further emphasizing not only how those animating and those animated force each other to act, sharing the action through the act of animating, but also how there is an added layer in anime animation: that the figurative acting codes must be adapted, something outside both the animator and animated, forcing them both within a certain mode of expression. Indeed, as if to comment on this and highlight the meta-performance, the soft-spoken junior animator Yasuhara is curious of Iguchi's work, and while peering over her shoulder loudly knocks over the garbage can. Yasuhara's face immediately switches to rounded, white-eyes, a figurative code often used to display shock, and in this case, embarrassment. However, Iguchi does not take any notice as she is concentrating so hard on her drawings.

The shared (and thus external, non-individualized) nature of figurative expressions is brought into attention throughout this episode. When Miyamori looks at some other key-frames in episode 3 , the characters have certain conventional facial expressions on them, which we view by seeing Miyamori flip through each image, the camera focusing on the papers. The next cut is to Miyamori with another separate, conventional facial expression on her face as she remarks with a sigh how cute the characters are. Later in the episode, when scared about a possible error, Miyamori makes the round, white-eyed expression for mortified shock that Yasuhara did when she knocked over the garbage can (though without a blue tint, this time). Finally, at the end of the episode, both Yasuhara and Miyamori sit and watch the final cut of the sakuga of the animated, pained expression.

After Iguchi finishes the key-frames with the new expression, they are sent to Ogasawara, the general animation supervisor. In one sequence, she reviews the frames and adds one single line to one frame, explaining to Yasuhara how just that one line to the outline of the character's chin can make a difference. In this sense, even the key-animated frames receive an adjustment, a masterfully placed line that changes the movement of the expression, another agential layer added to the act of animation. Following this, the images are taken to be colored and are then edited together to make the final cut. As such, there are multiple people involved in just this one sequence, where actions that effect the final product are traceable not only from person to person but also through the enactment of the figurative code for that pained expression. This episode, in particular, displays how many people are involved as agents in the production process, but it also reveals how crucial anime's media-form is, something external to the animators, one carefully practiced through repetition of references. In these sequences, because they are already performed in anime's media-form, the practice becomes emphasized as they are referencing themselves as anime.

To close off this section on media-form, it is worth noting how anime's media-form has increasingly become associated with Japan, shifting from a niche product to a media-form that represents Japan as a nation, globally. This is subtly revealed in one of the conversations that occurred during the above-mentioned conflict between the 2D animator (Endō) and the 3D animator. In episode 5, Endō and another animator remark on how 3D animators should just go to California to produce such flavorless animations, inciting a difference between $3 \mathrm{D}$ and $2 \mathrm{D}$, where $2 \mathrm{D}$ is connected to anime (and more suited to anime). This statement occurs during a drinking session with Saburō Kitano, a character (modeled after animator Ichirō Itano) who teaches "Japanimation" to 3D animators. It is important to note that this is the first time that the term "Japanimation" was used in the series (generally using the word "anime"), a term that nationalizes the animation technique and media-form. Playing the role of the wiser, senior animator, Kitano notes how he teaches them how to distort time and the images using the 3D software, and that, though the 3D animators cannot draw, they love "anime"

3 This was actually noted by a Japanese fan on Twitter (HASSO 2017), who provided images to display the comparison and noted the hard work involved in their respective productions. 
(connecting "Japanimation" with "anime"). Ultimately, he proposes that they work with 3D animators, cooperating with them, learning the capabilities and difficulties of 3D animation to improve anime's quality. He acknowledges that the differences in materials and technology provide different ways to animate, each with various strengths and weaknesses, and this negotiation between these two can be productive, helping to make better quality anime. Here, we see an acknowledgement of the clashes between 3D and 2D animation and the different capacities of their technologies, as well as a connection between them that is through learning the "basics of Japanimation", with Kitano stating that techniques and "an (artistic) sense" (which is implied is something that can be taught and learned, not natural) of 2D animation can be useful for 3D animation. There is an implicit understanding that there is a difference between anime's media-form and other types of animation, even if it is here revealed in relation to a "national" distinction.

\subsection{Transnationality}

While the relation of anime to Japan is very strong, anime works have been transnationally consumed since Tetsuwan Atomu in the early 1960s (Mihara 2014), and portions of anime's animation have been outsourced throughout Asia since the late 1960s (Choo 2015). This transnational production system increased in the 1970s and 80s (Choo 2015; Kim 2015), continued into the 2000s (Masuda 2016), and still occurs on a large scale to this day. Not only related to animation, increasingly, funding is coming from China and the US (often to animate franchises, the intellectual property of which is owned by those funding the production), and globally available online streaming services are supporting (and promoting) anime productions that will be exclusive to their services (Sudo 2017). It is in this context that the agency of the labor-here defined as the capacity to make decisions that affect the final product—of anime production takes on another geopolitical dimension: who, in which country, is really making the decisions? Because of our inability to work through transnational productions without relying on national frameworks, there is a tendency to legitimize anime as "authentic" due to its relation to Japan ("it is mostly a Japanese production, making it Japanese"; "the Chinese are just providing the funding for this"). Conversely, such reliance on national frameworks can be exploited to show the power of a non-Japanese market over Japanese production ("they are catering to our tastes now"). Either of these readings are possible, because there are few alternative frameworks ${ }^{4}$ to engage with when we consider anime's layers of transnationality in its production, distribution, and consumption.

Much of the transnational production in anime occurs through sub-contracting labor from the major studio that is tasked with producing the anime. While Shirobako depicts a generally positive view of freelancing through the almost mentor-like role that the freelance animator Misato Segawa plays for Miyamori, the series provides a less favorable view of sub-contracting for animation. This is done through sequences involving a sub-contractor called Studio Titanic, portrayed as a run-down office with sloppy organization and poor production quality. What the series leaves out is the large-scale reliance on such subcontractors and freelancers in the industry, and how much of this actually has a transnational component to it.

Indeed, this is true of Shirobako itself. The series is produced by the Japanese studio P.A. Works and Warner Entertainment Japan (a subsidiary of the U.S. company). Beyond the funding, there are other laborers involved in the production as well. This includes work such as finishing animation (usually the coloring), which was partly outsourced to YABES, a studio in South Korea, and TAP (Toei Animation Philippines) in the Philippines. Even key animation was done in South Korea, for example, in episode 13 which used YABES and Hanil Animation studios there. Some of the backgrounds

4 One of them may be regionality, though it may not always follow established geographies. For a recent approach to media regionality, see Marc Steinberg and Jinying Li's recent work on media platforms and region (Steinberg and Li 2017) and Thomas Lamarre's work on media geographies (Lamarre 2015). 
in episode 3 were done by Studio Suu, with Vietnamese names credited at the end of the episode. Other roles were filled by non-Japanese as well, such as animation directing, which for episode 21 was done by Rong Hong and Jung-Duk Seo (which were presumably done in Japan but could have occurred in South Korea). For episode directors, along with Japanese directors Fumihiko Suganuma (episodes 7, 13, and 15) and Hideaki Kurakawa (episodes 12, 16, and 22), South Korean Jong Heo directed multiple episodes (episodes $3,8,17$, and 24). Storyboarding was also done by Heo for episodes $3,8,10,17,23$, and 24 (most probably in Japan). This means that some of the key sequences that were analyzed above, specifically in episodes 3 (the facial expressions) and 23 (the issues between manga and anime; the complex "boss fights"), are partially due to the labor of Heo, who worked as episode director and/or on the storyboard (which itself becomes a transnational actor). Thus, there is an important element of agency that is transnational, not just in the animation but in other levels of production, contributing to the labor in ways that bare it on the final anime product.

Because much of this information is gleamed from the credits, it is difficult to determine exactly which sequences were done by which animator (for Japanese and non-Japanese staff), and besides the studio name, it is difficult to place exactly where the labor took place (inside or outside of Japan). That aside, one can see that the dispersal of agency is one that is not just confined within the borders of Japan but operates across anime's transnational production network. This significantly complicates the conception of anime as an address from Japan to Japan. With this in mind, we cannot forget that, according to Shirobako, within Japan there are assertions of agency at multiple levels in the production hierarchy, so this could also be applied to those working outside of Japan (and those from outside of Japan working within Japan). This is also true for the impositions, the limits on agency that occur in the process of production: the working through, with, and on materials and technologies that act upon the animators; the media-form which comes from outside of the animators, which they must learn, through copying and (re)production in iterative performances.

The above-described conception of anime's performance that highlights the importance of repetition diverges considerably from the prevailing conceptions of creative labor. The "standard" take on creative labor presumes a "top-down" type of creativity, in which, according to Joon Yang Kim, there is a valorization of intellectual labor over physical labor, in which directing is considered more important than the physical labor of animating (2015). Here, the creative visionary of the director is the esteemed agent, their instruction the authoritative guidance from which orders flow, which are merely "followed" by the animators (or other staff) — the director is the author's voice, the agent making the address, producing a commentary (often on Japan) through the process. Such an approach is an implicit endorsement of a type of creativity that is based on a modernist reverence for novelty in the act of creation (Pang 2012), a valorization of departure from trend rather than the "following of orders" and repetition, which are denigrated as corollary to (mass) industrialized production. An alternative view of creativity is beyond the scope of this essay, ${ }^{5}$ but for the time-being, I would suggest that a conception of dispersed agency, one in which all parties involved have different degrees of power in this hierarchical structure, allows us to see a more collaborative, transnational process come into play in anime production. This is a similar mode of viewing that is also practiced by sakuga-fans.

\section{Sakuga-Fans: Activities and Effects}

\subsection{Focus on Anime's Media-Form}

As noted prior, throughout the series, Shirobako directly highlights sakuga animation (e.g., scenes of flipping through key-frames, additions at the end of the episode), forcing a particular type of consumption upon the viewer, making them consume not just a narrative about production that

5 I discuss this in more detail in a forthcoming book chapter "Repeating Anime's Creativity across Asia" in Trans-Asia as Method: Theory and Practices. Edited by Jeroen de Kloet, Yiu Fai Chow, and Gladys Pak Lei Chong. London: Rowan and Littlefield International. 
focuses on the labor "behind" anime but also to acknowledge the actual animation itself, in its sakuga condition. This is the same focus for sakuga-fans, except it is expanded to their engagement with any anime work. In other words, they are explicitly concerned with anime's media-form, specifically from a technical, production process, and aesthetic perspective. This is evident in the works that sakuga-fans produce, published on the internet. Emblematic of such works is the popular Sakuga Blog, run by a multinational group of English-speaking fans: (using the online pseudonyms of the top 3 writers) $\mathrm{kViN}$ is located in Barcelona, Spain; Disgaeamad is located in Tokyo, Japan; and liborek is located in the Czech Republic. Sakuga Blog publishes interviews with animators (conducted by the site or translations from Japanese publications into English, such as a translation of an interview with Megumi Kouno, Disgaeamad 2016), reviews of the production of airing TV shows, and runs a large archive of animation sequences. This archive is called Sakuga Booru, which hosts short sequences of animation (without sound) that are organized and categorized according to anime title and the key-animator that it is credited to. With each animation tagged with that information, the site is easily searchable by either anime title or animator. There are also a number of anime YouTubers that focus on sakuga. They produce videos where they discuss specific animators, showing certain segments, often estranged from their context in the narrative, detailing the production history of that work and/or describing the history of the animators and their work. Some of them have substantial followings, such as AnimeAJay from the UK, who has 136,000 subscribers. While there are other resources by and for fans of sakuga, I will be focusing on these here.

It is important to note that these fans are explicitly interested in anime's media-form and by extension its production process, and through this they become somewhat critically engaged, mainly in concern to the terrible work conditions of the animators themselves. This is something left out of Shirobako, which does not show the dire situation of many of the animators. While it does display the stresses involved in the demanding work and portrays some animators (and other staff) as less financially stable, the series makes no sustained remarks in the narrative on the severity and precarity of their livelihood, or the meager pay of the animators. This is a well-known issue in the anime industry, something that many fans have taken note of, and sakuga-fans are actively critical of the situation. This is particularly evident in the blogs, which decry the animators' pay and work conditions. Such a position is blatantly evident in a recent post on Sakuga Blog entitled "The Struggle of Anime's Novice Creators", which goes into great detail about the difficulties of junior animators, the harsh working conditions, low pay, and poor job security, but also suggests possible alternatives and praises studios like Kyoto Animation, which does seem to provide a favorable (and successful) model for other studios (kViN 2018a).

In addition to the direct discussion of their work situation, one might also interpret the focus on animators as an attempt to address this. Sakuga Blog highlights newcomers in the industry, who are in the most precarious position as junior animators, making the least amount of money, lacking experience and contacts. Though not explicitly stated, this praise and promotion works as a way to elevate their status (especially among English-reading fans) and could potentially raise their profile within the industry. As such, sakuga-fans are to a degree politically active, acknowledging the poor working conditions and pay of the animators, actively investigating and disseminating information on the situation, and, in a sense, calling for solutions to these systemic problems. It is their engagement with the media-form that then involved research into the production process, which exposed the uglier side of the industry. They are also keenly aware of the importance of sub-contracting in the industry, even using an image from Shirobako's fictional sub-contractors Studio Titanic in their blog post on the role of outsourcing ( $\mathrm{kViN} 2018 \mathrm{~b})$. Accompanying this understanding of the integral role of outsourcing in anime production has also led to an acknowledgement of anime's transnational production, which I will detail below. 


\subsection{Engaging Anime's Transnationality}

Many of the activities of sakuga-fans function as a form of knowledge production. This includes the blogs that hosts interviews, the videos that expose (and educate) other fans on animators and the production process, and the archiving work that categorizes animators with the sequences they animated. This is an extremely difficult research process, as the credits of an episode do not include which animator drew which sequence. Sakuga YouTuber AnimeAJay, from the UK, describes his method of determining which animators produced which scenes in an extended video (AnimeAjay 2017). He explains how he carefully combs through the credits, cross-referencing the staff of each episode with online information of what projects and episodes the animators and animation supervisors worked on in the past. Then, he starts to compare parts of the episode to examples of their previous work. He looks for certain similarities of parts of the characters, such as the ears or the noses, or how different animators draw explosions, smoke, or the crumbling rock formations that are typical in action anime. ${ }^{6}$

Such a practice of viewing would align with what Lamarre sees in anime's visuality, that is, a multi-layered "field dense in information" (Lamarre 2009, p. 145). Lamarre explains that through this type of viewing, "character design or mecha design may prove more important than story or character, or the key animation of battle scenes may garner as much attention as character development" (p. 145). Lamarre explains that this mode of viewing "flattens the hierarchy of production by which directors are supposed to be of primary importance, followed by producers or writers, followed by animation directors, key animators, and character designers" (p. 145). In this sense, building on Lamarre's observations, we can see that sakuga-fan practices intimately engage with the media-form of anime, flattening received hierarchies of production. This allows for the appreciation of anime as a performance of animation, one that does not necessarily have to be seen as exclusive to Japan. As such, I would suggest that when fans search out the sequences by particular key-animators, they are looking for exceptional performances of anime's media-form rather than seeing anime as a commentary on Japan (or Japanese society).

There is thus an effect of a levelling of the transnationality of anime's production in the knowledge produced by the sakuga-fans, in which they do not focus on anime's Japaneseness. This is not necessarily explicitly said but is the effect of the way in which they position non-Japanese animators. While this is present in the archive of Sakuga Booru, which features non-Japanese animators such as Austrian Bahi JD in the same manner as Japanese animators, there are more active approaches that integrate non-Japanese animators as valued instances of anime performance. For example, the Sakuga Blog, conducted a long interview with Korean animator Se Jun Kim, discussing his recent work as a director and his accomplishments as an animator (Kraker2k 2017). This is also present in the YouTube community. Anime YouTuber (that is, not specializing in sakuga, but talking about anime generally) The Canipa Effect began a series called "animator spotlight", which discusses a particular animator's work across different anime with Kim as the focus (The Canipa Effect 2015). The very first animator he chose was not Japanese (but works in Japan), and he labels him the "Modern Mecha Master", adoringly describing his work, and only mentions his ethnicity once, stating that as "a Korean animator in Japan, he hasn't lent his skills to a huge amount of series, but the ones he has have certainly been noticeable." From his description of the scenes shown, it is very clear that it is his skill as an animator that is the focus, in performing mecha animation sequences that are thrilling, stating "it is people like Se Jun Kim that really do make mecha anime a visual spectacle." The Canipa Effect focuses on animation performance and, as such, equalizes Kim along with the other Japanese animators he discusses in later videos in the animator spotlight series.

6 AnimeAJay focuses on Dragon Ball Super, which continues a tradition of certain tropes, such as the crumbling rocks in power-up sequences. 
Such an equalizing through juxtaposing animators next to one another is done in other sakuga-fan videos. A YouTube channel that focuses on sakuga, BlueSakuga, features videos that are the sequences done by one particular animator with no commentary. Though only having 11 videos, two of those are on South Korean animators (Se Jun Kim and Sunghoo Park), putting them alongside other videos of Japanese animators such as Masuda Hirofumi and Nakamura Yutaka (BlueSakuga 2015c, 2015b, 2015a). This is also done in the "animation breakdown" video of episode 130 of Dragon Ball Super (2015-18) by YouTuber AnimeAjay (2018). Here, he comments on part of the production process, displaying and crediting the episode directors and the different sequences to their respective key-frame animators, and notes who the specific animation supervisors were. In this particular Super episode, which was widely praised for its high quality of animation and as the climax of the series, AnimeAJay goes through each complex action animation sequence, spending over $18 \mathrm{~min}$ describing multiple sequences that are each only a few seconds long. Briefly describing the animators' histories in the show, he juxtaposes Chinese animator Yong-ce Tu next to other Japanese animators. He describes how he was a 2nd key-animator since his introduction in episode 34 until episode 90, in which he drew 100 cuts for the major fight in that episode and provided key-animation (and some 2nd key-animation) from that point onwards. He describes how he has learned from Naotoshi Shida and Yuya Takahashi and then positively describes the sequence by Tu. He thinks that Tu is also a fan of Dragon Ball Z (1989-96) as the parts of the fight he animates feature "flurries straight out of the [fights from] the Cell and Buu [story-]arcs." He praises the exaggeration of the figures in the Dragon Ball Super fight, saying that it goes "hand-in-hand" with Tate Naoki's animated sequences earlier in the episode. He also notes how one particular sequence is a repurposed (completely redrawn, but maintaining the key-frames for the movement and storyboard) sequence by Takahashi, but this time it is done by Tu. He then moves on to describe the episode director, Ryōta Nakamura, and how it was from his storyboard that much of the action came. He notes how even still sequences are well composed, describing how images of the background give the audience time to breathe between intense action sequences. Afterwards, AnimeAJay analyzes the work of the Japanese animators in the same amount of detail, including a sequence by Shida, describing the movement that he feels is characteristic of his character animation (the shaking head of the character as he powers up) and noting how impressive the details on the characters are in their stills, while highlighting the complexity of the movement involved.

In his videos, AnimeAJay pays careful attention to the animation, describing the vibrancy of colors, the contortion of bodies, and the impact of certain images, praising the rhythms between movement and stills. In these analyses, there is attention paid to the mark of individual performances while acknowledging the need for consistency. He also highlights the multiple actors that are involved in anime production and their distinctive contributions, while still grasping the chain of command. Fan works such as this acknowledge the transnationality of anime and the multitude of actors involved, understanding the difficulties of working with the medium of animation. Here, just as in Shirobako, the individual contributions are underscored by the clear recognition of the dispersal of agency, even across national boundaries, through intensive engagement with anime's media-form.

\section{Consuming Production: Towards Rethinking Approaches to Transnationality}

Not isolated to anime, there is an increasing need to reconcile the multiple layers of transnationality and dispersal of agency in the production, distribution, and consumption of global media in general. With agency dispersed across a network, one which occurs across national borders, transnationality fundamentally shifts conceptions of the "local" and "global": a "local" site of production may be part of a larger production network that crosses extensive distances and national boundaries, intersecting with the "global" at that site. Following Latour, when we trace these transnational networks whatever a (national) "society" is can be understood as differently assembled, and as media like anime are so often tied to notions of locale-based culture (anime as a commentary on Japanese society), we must rethink how "cultural production" can be considered in light of these cross-border assemblages. This also means that the material conditions of labor can have diverse 
affects, even for related media. For example, manga (made with a minimum of one human agent in one locale) $)^{7}$ and anime (made with multiple studios and sub-contractors in various locales) can have different types of transnationality in terms of production. Similar divergences may also be revealed in "actor-network" analyses of consumption. However, as we unravel the specific dynamics of these media, we may also find clues to alternative modes of engagement that move beyond the inter-national and consumption-production dichotomies.

In the above examples, one can see how "consumption" is also tied very distinctly to "production." Just as Shirobako provides an anime about production to be consumed, sakuga-fans produce works from their own consumption practices; they consume the production processes of anime (not just its results) and produce writing, archives, and videos on this information. This process often takes on a transnational component that reveals a particular type of transnationality. In sakuga-fan blogs and videos, we can see how anime's transnationality is multilayered, with an understanding of the outsourcing occurring for anime's animation and the consumption of anime in places outside of Japan. At the same time, there is an acknowledgement of non-Japanese working in the industry and producing fan products (like the blogs) inside of Japan. As such, the transnational in regards to anime is not something that is clearly outside of Japan but includes Japan as well as other countries. This is an important shift to consider, a type of flattening that goes across national borders, an alternative to an inter-national inside-outside of Japan dynamic (anime as a commentary on Japan; Japanese culture consumed abroad) when we conceptualize anime's transnationality. It is sakuga-fans' consumption of production and their own subsequent transnational production that highlights the dispersed nature of decision-making and contributions to anime's production and the multilayered transnationality of anime. Sakuga-fans practice an approach that delves into the mechanics of anime's media-form, focusing on skilled performances and exploring the labor behind them, acknowledging the multiplicity of actors involved, opening up anime to its transnational potentials in their engagement with both Japanese and non-Japanese animators. Globally popular, anime's images tend to signal Japan, but sakuga-fans recover the transnationality of anime's production by engaging with its media-form.

Funding: This research received no external funding.

Conflicts of Interest: The author declares no conflict of interest.

\section{References}

AnimeAjay. 2017. How to Identify Animators' Styles in Dragon Ball (and More!). Available online: https://www. youtube.com / watch?v=_xd7cyPoG0k\&list=PLnrgbq-d2KQEZ6D1_J2lfN1IcGqDjDKuN\&index=6\&t=0s (accessed on 8 May 2018).

AnimeAjay. 2018. The Greatest Showdown! Episode 130 Animation Breakdown—Dragon Ball Super. Available online: https:/ / www.youtube.com/watch?v=otoPMI7NRD4\&list=PLnrgbq-d2KQEZ6Dl_J2lfN1IcGqDjDKuN\& index $=5 \& \mathrm{t}=401 \mathrm{~s}$ (accessed on 8 May 2018).

Azuma, Hiroki. 2009. Otaku: Japan's Database Animals. Translated by Jonathan E. Abel. Minneapolis: University of Minnesota Press.

BlueSakuga. 2015a. 中村豊 (Yutaka Nakamura) MAD. Available online: https://www.youtube.com/watch? v= BGqGuQFaDhw\&t=31s (accessed on 8 May 2018).

BlueSakuga. 2015b. 朴性厚 (Sunghoo Park) MAD. Available online: https://www.youtube.com/watch?v= bEDHAeg_vc\&list=PLnrgbq-d2KQEZ6D1_J2lfN1IcGqDjDKuN\&index=2\&t=0s (accessed on 8 May 2018).

BlueSakuga. 2015c. 金世俊 (Se Jun KIM) MAD. Available online: https://www.youtube.com/watch?v= 6mcw9ucnHug\&list=PLnrgbq-d2KQEZ6D1_J2lfN1IcGqDjDKuN\&index=3\&t=0s (accessed on 8 May 2018).

7 Latour asserts a methodology that stringently "flattens" site-specific (what he calls "local") and macro-conceptions (what he calls "global"), and, indeed, the materials, tools, and media-form of manga extend beyond the confines of the site of production. However, I am (over)simplifying this here merely to highlight how anime and manga's differences in materials, tools, and media-form can lead to important divergences. 
Choo, Kukhee. 2015. Hyperbolic Nationalism: South Korea's Shadow Animation Industry. In Mechademia 9: Origins. Edited by Frenchy Lunning. Minneapolis: University of Minnesota Press, pp. 144-62.

Condry, Ian. 2013. The Soul of Anime: Collaborative Creativity and Japan's Media Success Story. Durham: Duke University Press.

Crafton, Donald. 2013. Shadow of a Mouse: Performance, Belief and World-Making in Animation. Berkeley: University of California Press.

Disgaeamad. 2016. The IDOLM@STER Cinderella Girls Staff Interviews-Megumi Kouno. Sakuga Blog (Blog). August 22. Available online: https://blog.sakugabooru.com/2016/08/22/the-idolmster-cinderella-girlsstaff-interviews-megumi-kouno/ (accessed on 5 May 2018).

HASSO. 2017. LWAとSHIROBAKOの共通点について、LWA第6話ラストのアッコの表情動かし方を観て て、SHIROBAKO第3話の作画修正回のラストと何となく重なった。このカットもきっと並々ならぬ拘 りと沢山人の苦労があって描かれたのだろうなあ.... Tweet. @shimesaba_taro (Blog). February 15. Available online: https:/ / twitter.com/shimesaba_taro/status/832059173709901825 (accessed on 2 May 2018).

Iwabuchi, Kōichi. 2010. Undoing Inter-National Fandom in the Age of Brand Nationalism. In Mechademia 5: Fanthropologies. Minneapolis: University of Minnesota Press, vol. 5, pp. 346-48.

Kim, Joon Yang. 2015. South Korea and the Sub-Empire of Anime: Kinesthetics of Subcontracted Animation Production. In Mechademia 9: Origins. Edited by Frenchy Lunning. Minneapolis: University of Minnesota Press, pp. 90-103.

Kraker2k. 2017. Exclusive Interview with Sejoon Kim-Director of Gundam Twilight Axis. Sakuga Blog (Blog). September 7. Available online: https:/ /blog.sakugabooru.com/2017/09/07/interview-with-sejoon-kim/ (accessed on 5 May 2018).

kViN. 2018a. The Struggle of Anime's Novice Creators. Sakuga Blog (Blog). March 7. Available online: https: / / blog.sakugabooru.com/2018/03/07/the-struggle-of-animes-novice-creators / (accessed on 5 May 2018).

kViN. 2018b. Keeping the Anime Industry Afloat from the Shadows: Wanpack and Outsourcing Studios. Sakuga Blog (Blog). April 26. Available online: https://blog.sakugabooru.com/2018/04/26/keeping-the-animeindustry-afloat-from-the-shadows-wanpack-and-outsourcing-studios/ (accessed on 5 May 2018).

Lamarre, Thomas. 2009. The Anime Machine: A Media Theory of Animation. Minneapolis and London: University of Minnesota Press.

Lamarre, Thomas. 2015. Regional TV: Affective Media Geographies. Asiascape: Digital Asia 2: 93-126. [CrossRef]

Latour, Bruno. 2005. Reassembling the Social: An Introduction to Actor-Network-Theory. Oxford: Clarendon.

Masuda, Hiromichi. 2016. Dejitaru ga kaeru anime bijinesu (デジタルが変えるアニメビジネスーAnime Business, Changed by Digitalization). Tokyo: NTTshuppan.

Mihara, Ryōtarō (三原龍太郎). 2014. Kūru Japan wa naze kirawareru no ka: “Nekkyō" to “reishō" wo koete(ク一ル・ ジャパンはなぜ嫌われるのかー「熱狂」と「冷笑」を超えて—Why Is CoolJapan Hated? Beyond 'Enthusiasm' and 'Cynicism'). Tokyo: Chuo Koron Shinsha.

Pang, Laikwan. 2012. Creativity and Its Discontents: China's Creative Industries and Intellectual Property Rights Offenses. Durnham and London: Duke University Press.

Steinberg, Marc. 2012. Anime's Media Mix: Franchising Toys and Characters in Japan. Minneapolis: University of Minnesota Press.

Steinberg, Marc, and Jinying Li. 2017. Introduction: Regional Platforms. Asiascape: Digital Asia 4: 173-83.

Suan, Stevie. 2017a. Anime No 'Kōisha': Animēshon Ni Okeru Taigenteki/Shūjiteki Pafōmansu Ni Yoru 'Jiko' (「アニメの「行為者」ーアニメーションにおける体現的/修辞的パフォーマンスによるーAnime's Actors: Constituting 'Self-Hood' through Embodied and Figurative Performance in Animation). Animēshon Kenkyū 19: 3-15.

Suan, Stevie. 2017b.t Anime's Performativity: Diversity through Conventionality in a Global Media-Form. Animation: An Interdisciplinary Journal 12: 62-79. [CrossRef] 
Sudo, Tadashi (数土直志). 2017. Dare ga korekara no anime o tsukuru noka: Chugoku shihon to netto haishin ga okosu shizuka na kakumei (誰がこれからのアニメをつくるのか? 中国資本とネット配信が起こす 静かな革命—Who Will Make Anime in the Future? The Quiet Revolution Caused by Chinese Capital and the Internet Distribution). Tokyo: Seikaisha.

The Canipa Effect. 2015. Se Jun Kim |Animator Spotlight| "Modern Mecha Master". Available online: https: / / www.youtube.com/watch?v=zekjRSjFdKM\&index=7\&t=0s\&list=PLnrgbq-d2KQEZ6Dl_ J2lfN1IcGqDjDKuN (accessed on 6 May 2018). 